\title{
Erratum to: Comparison of diagnostic performance between single- and multiphasic contrast-enhanced abdominopelvic computed tomography in patients admitted to the emergency department with abdominal pain: potential radiation dose reduction
}

\author{
Shin Hye Hwang • Je Sung You • Mi Kyong Song • \\ Jin-Young Choi • Myeong-Jin Kim • Yong Eun Chung
}

Published online: 19 December 2014

(C) European Society of Radiology 2014

\section{Erratum to: Eur Radiol}

DOI 10.1007/s00330-014-3481-6

Due to a transcription error, the affiliation for Je Sung You in the article entitled "Comparison of diagnostic performance between single- and multiphasic contrast-enhanced abdominopelvic computed tomography in patients admitted to the emergency department with abdominal pain: potential radiation dose reduction", was incorrectly published. We would like to clarify that the affilitation should read as follows:

Department of Emergency Medicine, Yonsei University College of Medicine, Seoul, Korea

We apologise for this error in the author list; the error was multifactorial.

The online version of the original article can be found at http://dx.doi.org/ 10.1007/s00330-014-3481-6.

S. H. Hwang · J.-Y. Choi • M.-J. Kim • Y. E. Chung $(\bowtie)$

Department of Radiology, Research Institute of Radiological

Science, Yonsei University College of Medicine, 50 Yonsei-ro,

Seodaemun-gu, Seoul 120-752, Korea

e-mail: yelv@yuhs.ac

\section{K. Song}

Biostatistics Collaboration Unit, College of Medicine,

Yonsei University, Seoul, Korea

J. S. You

Department of Emergency Medicine, Yonsei University College of

Medicine, Seoul, Korea 